\title{
Using a simple equation to predict the microporation-enhanced transdermal drug flux
}

Alexey S. Rzhevskiy ${ }^{1}$, Krishna Telaprolu ${ }^{2}$, Yousuf H. Mohammed ${ }^{2}$, Jeffrey E. Grice ${ }^{2}$, Michael S. Roberts ${ }^{2,3}$, Yuri G. Anissimov $v^{4,1, *}$

1 Institute of Molecular Medicine, Sechenov First Moscow State Medical University, Trubetskaya 8, 119991 Moscow, Russia

2 Therapeutics Research Centre, School of Medicine, The University of Queensland, Translational Research Institute, Brisbane, Australia

${ }^{3}$ School of Pharmacy and Medical Sciences, University of South Australia, Adelaide, Australia

${ }^{4}$ School of Natural Sciences, Griffith University, Gold Coast, Queensland, 4222, Australia.

* Correspondence: email: y.anissimov@griffith.edu.au, phone: +617 55528496

\begin{abstract}
The mathematical model describing drug flux through microporated skin was previously developed. Based on this model, two mathematical equations can be used to predict the microporatio-enhanced transdermal drug flux: the complex primal equation containing a variety of experimentally-determined variables, and the simplified straightforward equation. In this study, experimental transdermal fluxes of three corticosteroids through split-thickness human skin treated with a microneedle roller were measured, and the values of fluxes compared with those predicted using both the more complex and simplified equations. According to the results of the study, both equations demonstrated high accuracy in the prediction of the fluxes of corticosteroids. The simplified equation was validated and confirmed as robust using regression analysis of literature data. Further, its capability and ease of use was exemplified by predicting the flux of methotrexate through the skin microporated with laser and comparing with published experimental data.
\end{abstract}

Keywords: Mathematical modelling; Microporation; Percutaneous penetration; Skin flux enhancement; Transdermal drug delivery 


\section{INTRODUCTION}

Due to its significant barrier properties, the stratum corneum (SC) presents major limitations to transdermal delivery of topically applied drugs. The barrier properties of SC, that are often described using a so-called brick and mortar structure, effectively restricts permeation of drugs molecules deep into the skin and underlying tissues [1, 2]. However, in the last two decades researchers have made a significant effort in investigating diverse chemical and physical enhancing techniques which lead to increased permeation of drugs through the skin, and revealing the potential of transdermal drug delivery as an important technique for therapeutic treatment and vaccination [3-6].

Microporation is one such physical technique that can be used to enhance transdermal drug delivery. The mechanism is based on the creation of micron-sized pores/holes in the SC before the application of a drug formulation, such as a cream, a gel, or a solution, etc. Such a technique can contribute up to 200-fold enhancement of transdermal permeation of drug molecules through the created pores/holes, compared to intact skin. If microporation is conducted gently, the resulting disruption of the SC causes no damage to the nearby tissues and nerve endings that are located in the dermis [7]. Further, the microporated SC barrier is restored within 1-2 days of being disrupted [8]. To date, several methods of microporation have been investigated: thermal ablation, radiofrequency ablation, electroporation, laser ablation and removable microneedles [7]. The technique of microporation is regarded as minimally or non-invasive, depending on application method, as well as being targeted and safe [9].

A major issue in drug delivery across microporated skin is that its rate cannot be easily predicted. This is a serious limitation for the microporation technique to be widely adopted into clinical practice. Recently, a new mathematical model for the prediction of drug flux across microporated skin was developed by Rzhevskiy, Guy and Anissimov [10]. This model resulted in a relatively complex equation for skin resistance that contained many parameters, including diffusion and partition coefficients of the drug in the vehicle and in skin, the thickness of the skin layers, etc. A simple equation to predict the flux was then derived from the complex equation, using approximations to parameters and various assumptions, and validated with experimental data obtained from the literature [10]. However, as there were many simplifying assumptions made for the derivation of the simple equation, in this study, we further validate the simple equation with the experimental data generated in our laboratory. Additionally, regression analysis was applied to further check the robustness of the simple equation. We compare the accuracy of predictions of the fluxes of three corticosteroids through the skin treated with a 
microneedle roller, using the simple and complex equations. In order to apply the complex equation, the transport parameters obtained experimentally in our previous work [11] were used, in addition to the parameters measured in this study. Further, the potential of the simple equation to be used in practical cases was discussed.

\section{MATERIALS AND METHODS}

\section{Theory}

The mathematical model, which describes permeation of drug flux from an aqueous vehicle through microporated skin, was previously developed [10]. The model was developed for the scenario when created pores/holes are distributed with a regular interspacing over the exposed area of skin. Based on this model, the flux $(J)$ through microporated skin was derived as:

$$
J=C_{v}\left[u\left(h_{v}-h_{f}\right) \frac{h_{v}-h_{f}}{D_{v}}+u\left(h_{s}-h_{f}\right) \frac{h_{s}-h_{f}}{K_{s} D_{s}}+\frac{h_{s c}}{D_{v} n_{p} \pi r_{p}^{2}}+\left(\frac{1}{D_{v}}+\frac{1}{K_{s} D_{s}}\right) \frac{l_{p}-2 r_{p}}{4 n_{p} l_{p} r_{p}}\right]^{-1}
$$

where $C_{v}, n_{p}, r_{p}$ and $l_{p}$ are the concentration of the drug in the vehicle, pore density, pore radius and the spacing between the neighbouring pores respectively. Additionally, $h_{s c}$ is the thickness of the SC, $h_{s}$ is the depth below the SC where concentration is assumed to be zero, $h_{v}$ is distance above the SC where drug concentration in the vehicle is assumed constant and $h_{f}$ $=\pi\left(l_{p}-2 r_{p}\right) / 8$. Further, $D_{v}$ and $D_{s}$ are the drug's diffusivities in the vehicle and viable skin, respectively, and $K_{s}$ is the partition coefficient of the drug between the viable skin and the vehicle. Finally, $u(x)$ is the unit step function $(u(x)=1$ if $x \geq 0$, and $u(x)=0$ if $x<0)$.

Eq1 for the flux through microporated skin was approximated as: [10]

$$
J \approx 0.36 n_{p} r_{p} M W^{-0.6} C_{v}
$$

where $M W$ is the molecular weight of the drug.

Importantly, it was necessary to apply several reasonable assumptions and approximations to derive the simple, convenient and straightforward Eq 2. Also, a number of assumptions was made during its validation with the experimental data obtained from different studies. Notably, the average radius of the pores for the case of microporation with microneedles was estimated from the needle's dimensions, that is a simplification and potentially a significant source of error. Overall, given the many assumptions, Eq 2 gave a good but not perfect accuracy of 
prediction, with the predicted values which varied within a factor of 5 of unity (i.e., $0.2<$ ratio < 5). Further, it was indicated that the accuracy of prediction was different for the drugs from diverse pharmacological groups.

In the current study, there were three major aims:

1. To compare the accuracy of prediction of drug flux through microporated skin, given by Eq 2 and the complex equation of the drug flux (Eq 1). It was also aimed to indicate whether the equations are applicable for the case of irregular interspacing of pores over the microporated area of skin as well as nonround shape of the pores. Skin microporation was performed with a microneedle roller. Three drugs from a group of corticosteroids were applied: hydrocortisone, desoximetasone and triamcinolone acetonide as there transport parameters in the dermis were previously established in our lab [11].

2. To perform statistical validation of the simple Eq 2 by regression analysis.

As the simple equation was derived with many assumptions on the value of parameters, most importantly that diffusion coefficient in the dermis is proportional to that in aqueous phase, it is reasonable to establish the derived equation's form robustness by using regression with the experimental data. The following regression equation was used:

$$
J=A n_{p}^{d} r_{p}^{b} M W^{-c} C_{v}
$$

where $A, b, c$ and $d$ are parameters to be varied in the regression analysis. According to the original Eq 2, these constants are $A=0.36, d=b=1$ and $c=0.6$.

Taking the logarithm of Eq 3, it can be linearised:

$$
\log J=\log A+d \log n_{p}+b \log r_{p}-c \log M W+\log C_{v}
$$

allowing linear regression to be performed, thereby guaranteeing that a global minimum is found. The regression analysis was performed using Scientist software.

3. To highlight the practical potential of Eq 2 in terms of its usefulness in the design of in vitro and in vivo experiments.

\section{Ethical approval}


The experimental part of the study was conducted under the ethical approval from Metro South Princess Alexandra Hospital (HREC/16/QPAH/064) and The University of Queensland Human Research Ethics Committee (2008001342).

\section{Chemicals}

Hydrocortisone, desoximetasone and triamcinolone acetonide were purchased from SigmaAldrich (Australia).

\section{Preparation of skin for the in vitro experiments}

Human abdominal skin segments for the experiments were obtained from patients undergoing abdominal plastic surgery. The skin was taken from three different female donors aged 47, 45 and 59 for the experiments with hydrocortisone, desoximetasone and triamcinolone acetonide, respectively. Adipose tissue was removed from the bottom part of the skin segments with surgical scissors and a scalpel, then the skin segments were stretched over and attached with pins to the rectangular wooden boards. The stretched skin was dermatomed with a Padgett Air Dermatome Model C (Integra LifeSciences Corporation, Cincinnati, USA). The desired thickness of dermatomed skin was set up at $500 \mu \mathrm{m}$ on the dermatome thickness scale, while the actual dermatomed skin thickness was measured with Vernier caliper by placing a piece of the skin, dermatomed from each segment, between two glass slides. The thickness of skin was calculated by deducting the thickness of two glass slides from the total thickness of two glass slides with a piece of skin between them.

\section{Skin microporation with microneedle rollers}

Each dermatomed skin segment was placed and stretched on a cork board that was covered by Parafilm. Subsequently, a microneedle roller (Fig.1) containing 540 microneedles in total, with the length of microneedles at $250 \mu \mathrm{m}$, was applied to the sections of the skin one $(1 \times$ rolled $)$ or two $(2 \times$ rolled $)$ times. For the second time application the microneedle roller was applied in the direction perpendicular to the first application. Fig. 2 shows the result of the microneedle applications for $1 \times$ rolled (Fig. 2 a,b) and $2 \times$ rolled (Fig. 2 c, d) skin sections.

To keep the force of microneedle roller application approximately constant, a cork board with the skin on it was placed on a flat surface kitchen balance. The force of the roller application was 
kept in a range of 4-5 $\mathrm{N}$ (400-500 g). A new microneedle roller was used for each set of experiments with a new drug, to ensure that the sharpness of microneedles, and consequently the depth of penetration and radius of all created pores remained approximately constant.

(a)

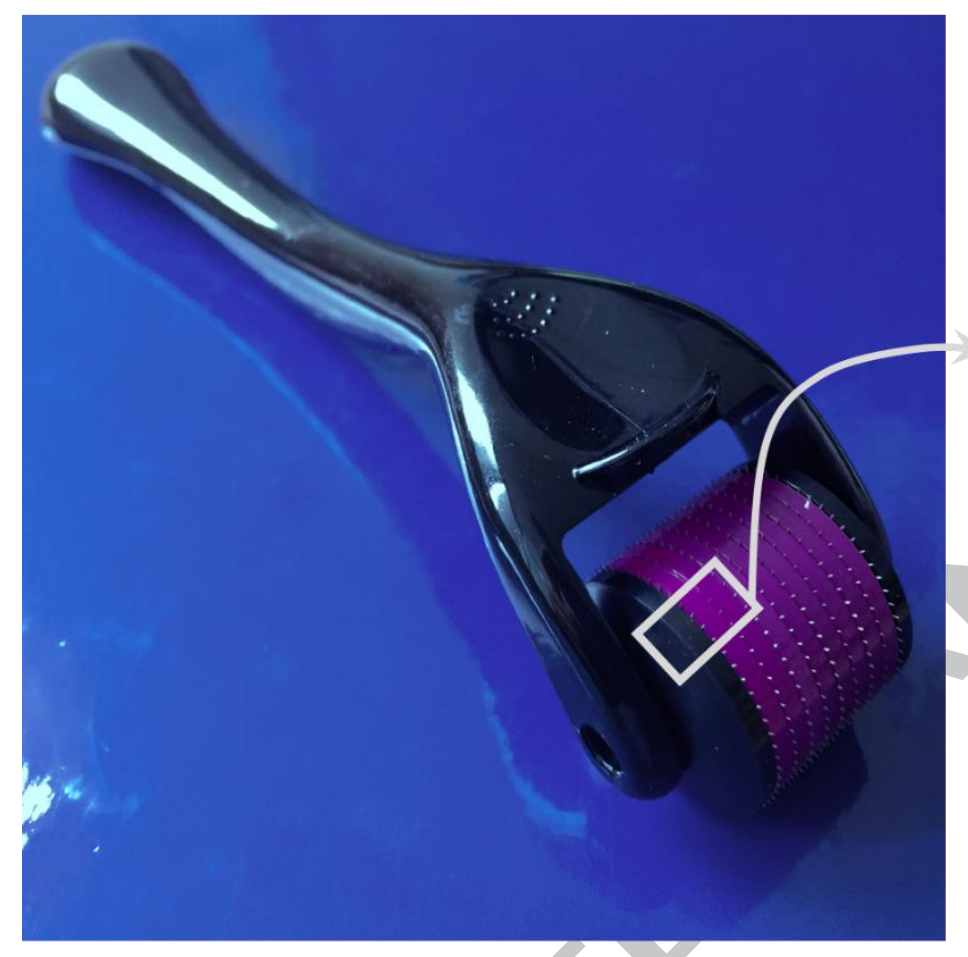

(b)

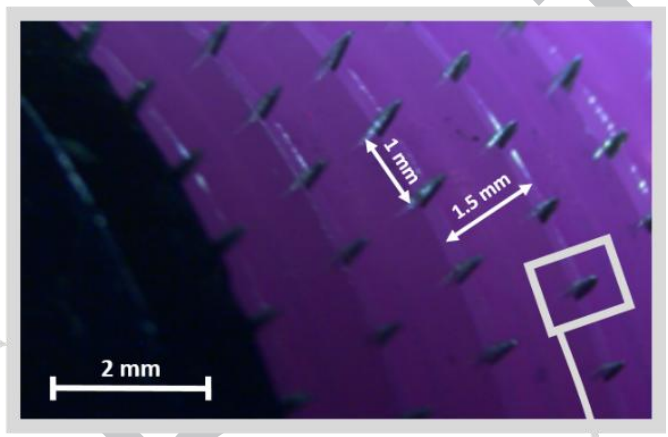

(c)

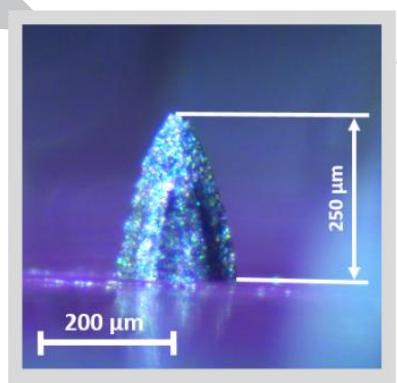

Fig.1. Microneedle roller and its microneedles.

\section{In vitro experiments}

The experiments were conducted using Franz cells with a diffusion area of $1.33 \mathrm{~cm}^{2}$. For the study with each drug four replicates were used to estimate drug flux through both $1 \mathrm{x}$ rolled and $2 \mathrm{x}$ rolled skin. The receptor chamber of each Franz cell was filled with PBS at pH 7.4. As the study was conducted over 48 hours, the receptor phase also contained sodium azide at the concentration of $0.05 \mathrm{wt} \%$ to prevent potential bacterial deterioration of the skin. The cells were placed in a water bath, equipped with a magnetic stirrer, at the constant temperature of $37{ }^{\circ} \mathrm{C}$. Prior to filling the donor chamber with drug solution, it was filled with $0.5 \mathrm{ml}$ of PBS and the skin was equilibrated for 30 minutes. After the equilibration, PBS was removed from the donor chamber with delicate tissues (Delicate Task Wipers, Kimtech®). Following that, the donor chamber of each cell was filled with $1.5 \mathrm{ml}$ of saturated PBS drug solution and covered with a sheet of Parafilm to prevent evaporation of the solution. The saturated solution of each drug was prepared by stirring an excess amount of drug in PBS at PH 7.4 for 24 hours at ambient 
conditions, and followed by filtration with a syringe through a $0.22 \mu 1$ polyvinylidene fluoride filter (Millipore Ireland ltd, Cork, Ireland). Samples of $200 \mu 1$ were taken from the receptor chamber at eight time points: $6,12,18,24,30,36,42$, and 48 hours after the beginning of experiment, and replaced with fresh receptor phase.

\section{HPLC analysis}

In order to analyze the samples, $80 \mu 1$ were taken from each sample and mixed with $20 \mu 1$ of an internal standard. The internal standards were: Triamcinolone acetonide for hydrocortisone and desoximetasone, and desoximetasone for triamcinolone acetonide. The analysis was performed by HPLC (Shimadzu, Kyoto, Japan) using a C18 column (Waters® Symmetry C18, $5 \mu \mathrm{m}, 150 \times$ $3.9 \mathrm{~mm}$ ) fitted with a guard column (Phenomenex® $\mathrm{C} 18,4 \times 3 \mathrm{~mm})$. An acetonitrile (ACN) water solution (40\% ACN : 60\% water) was used as a mobile phase at a flow rate of $1 \mathrm{ml} / \mathrm{min}$. The injection volume was set at $80 \mu 1$ and detection wavelength at $245 \mathrm{~nm}$. The retention times were 4.6, 5.7 and 2.8 minutes for triamcinolone acetonide, desoximetasone and hydrocortisone, respectively. The concentration of saturated solutions of drugs were estimated in the same manner after an appropriate dilution.

\section{Visualisation of created micropores, estimation of density and average radius of the pores}

A $2 \times 2 \mathrm{~cm}$ piece of female human abdominal dermatomed skin of $500 \mu \mathrm{m}$ thickness was placed on a firm backed cork board and derma roller was applied in a single roller action and a double roller action as described above in "Skin microporation with microneedle rollers" section. Rose Bengal dye which has a molecular weight of 974 was used to visualise the pores made by the derma roller. It has an advantage of not showing lateral transport in the pores due to its high molecular weight and therefore a relatively slow diffusion. Hence, only the dye in derma roller holes would be visualised. The dye at a concentration of $0.1 \mathrm{mg} / \mathrm{mL}$ was prepared in MilliQ water, and $200 \mu \mathrm{L}$ was added to the surface of the skin followed by gentle rubbing with a finger glove to ally it to the roller treated surface. The excess dye was wiped out from the surface using a dry Kimwipe (Kimtech Science ${ }^{\circledR}$ brand tissue paper). Images (Fig.2 (a) and (c)) were acquired using a Zeiss Stemi 2000-C optical microscope with a Zeiss Axiocam ERc 5s camera. A calibrated scale bar was used to measure the width and length of the pores using the AxioVision software (Release 4.8.2). 
In a parallel treatment regimen as described above after applying the rose Bengal to the derma roller treated skin, imaging was carried out by using MPM- FLIM. The imaging was performed at an excitation wavelength of $920 \mathrm{~nm}$ using the DermaInspect® system (JenLab GmbH, Jena, Germany) as previously described [12]. An ultra-short-pulsed, mode-locked, 80-MHz Titanium Sapphire laser (Mai Tai, Spectra Physics, Mountain View, California, USA) was used for excitation. The laser is tunable within a range of 710-920 nm and has an 85 fs pulse width. An objective lens C-Apochromat 10x/0.45 W (Carl Zeiss, Germany) was used and the space between the sample and the objective lens had a special water emersion lens solution for the (10x) magnification objectives. An excitation wavelength of $920 \mathrm{~nm}$ (two-photon) was used to excite Rose Bengal fluorescence. A constant incident optical power of $31 \mathrm{~mW}$, which is controlled at the rear of the objective, was used during imaging. Scattered laser light was suppressed by a $650 \mathrm{~nm}$ short-pass filter (Schott BG39 glass color filter) prior to reaching the FLIM detectors. The signal was further split into four wavelength intervals by dichroic beam splitters: 350 to $450 \mathrm{~nm}, 450-515 \mathrm{~nm}, 515$ to $620 \mathrm{~nm}$ and $620-650 \mathrm{~nm}$. The detection channel with 515 to $620 \mathrm{~nm}$ wavelength range was most suited for the Rose Bengal signal. The singlephoton pulses were collected by a time-correlated single-photon counting (TCSPC) SPC-830 module (Becker and Hickl [B\&H], Berlin, Germany), integrated into the MPT system. For each FLIM image, the acquisition size was $74 X 74 \mu \mathrm{m}^{2}$ with a scanning exposure of 13.4 seconds. The skin was imaged at the surface to identify the derma roller created needle holes (Fig.2 (e)). Images were acquired within 5 minutes of treating the skin with Rose Bengal post derma roller application. 


\section{ACCEPTED MANUSCRIPT}

(a)

(b)
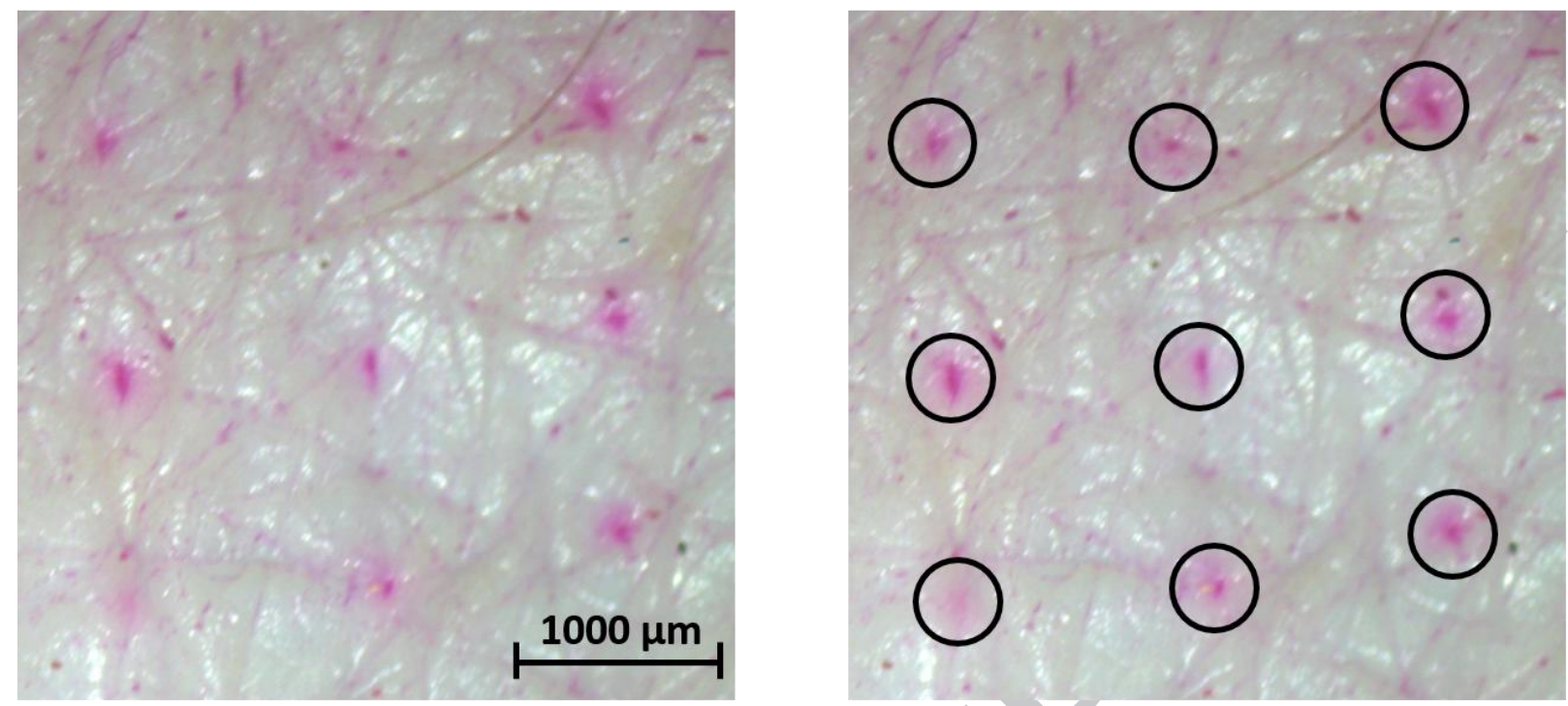

(c)

(d)

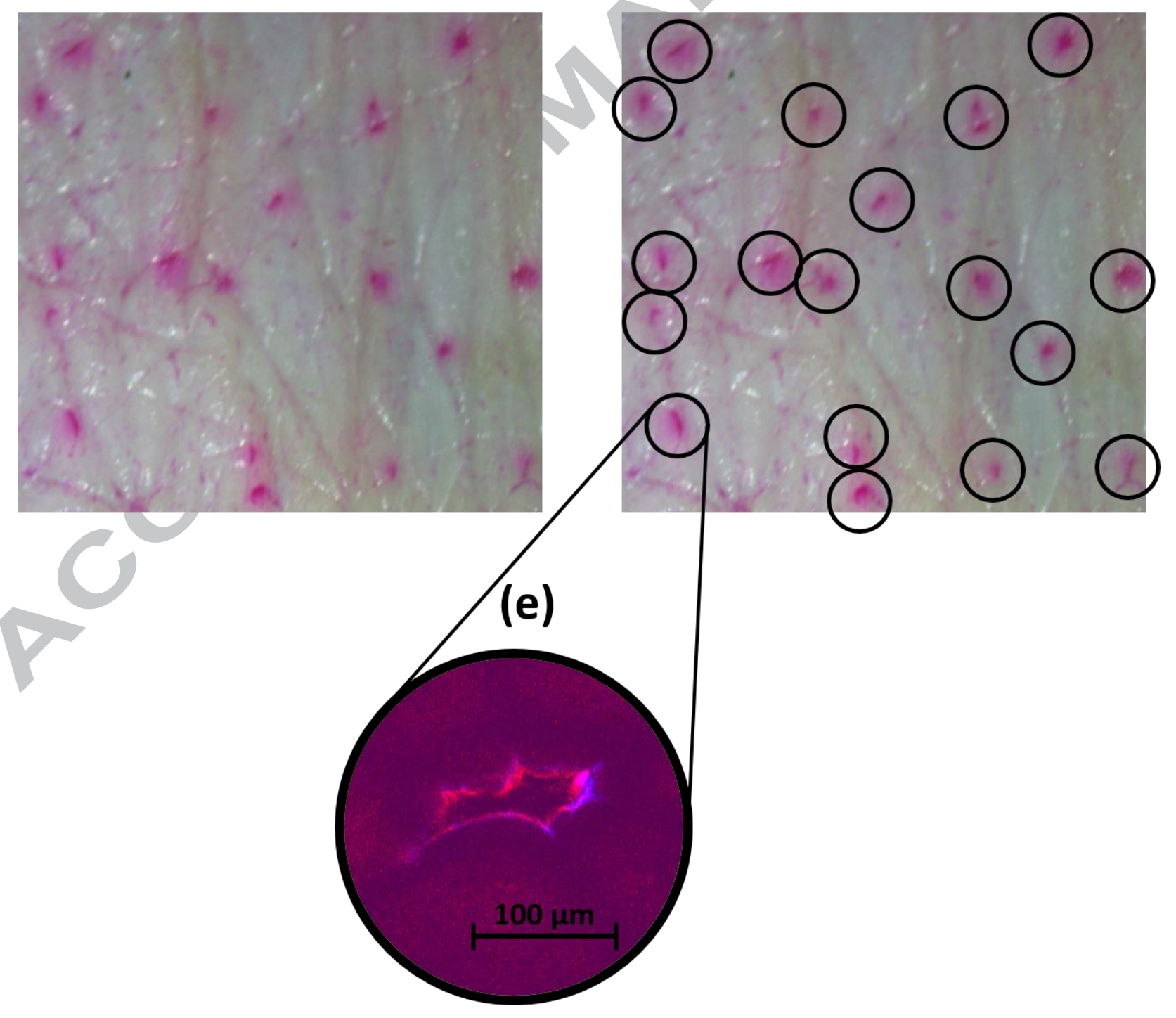


Fig.2. Images of the $1 \times$ rolled $((a)$, and the same image with pores highlighted by black circles (b)) and $2 \times$ rolled skin ((c), and the same image with pores highlighted by black circles (d)), with the micro-pores stained with Rose Bengal dye. The FLIM image of a single micro-pore (e).

\section{RESULTS AND DISCUSSION}

\section{Predicting the flux of corticosteroids}

The average thickness of dermatomed skin pieces, obtained from three different human donors, was indicated at $350 \mu \mathrm{m}$. Skin from single donor was used in the experiment for each corticosteroid. The values of cumulative drug amount in the receptor phase, estimated in accordance with the sample analysis performed with HPLC are demonstrated in Table 1.

Table 1 . The cumulative amounts of the drugs permeated through $1 \times$ rolled and $2 \times$ rolled skin.

\begin{tabular}{|c|c|c|}
\hline Drug & $\begin{array}{c}\text { Cumulative amount } \\
\text { at } 48 \mathrm{~h} \\
1 \times \text { rolled skin } \\
\left(\mu \mathrm{g} \mathrm{cm}^{-2}\right)\end{array}$ & $\begin{array}{c}\text { Cumulative amount } \\
\text { at } 48 \mathrm{~h} \\
2 \times \text { rolled skin } \\
\left(\mu \mathrm{g} \mathrm{cm}^{-2}\right)\end{array}$ \\
\hline Hydrocortisone & $19.2 * \pm 3.9$ & $36.4 \pm 3.5$ \\
\hline Desoximetasone & $3.8^{*} \pm 1.3$ & $9.8 \pm 2.4$ \\
\hline Triamcinolone acetonide & $1.27 \pm 0.16$ & $2.8 \pm 0.58$ \\
\hline
\end{tabular}

* The average total cumulative amount was calculated considering results of three Franz cells instead of four due to the impairment of one Franz cell during the experiment.

Fig. 3 shows that the trends of drug permeation across microporated skin versus time were linear for all three drugs. It is also apparent from Fig. 3 and Table 1 that drug permeation across $2 \times$ rolled skin is approximately two times higher than the permeation across $1 \times$ rolled skin for all three compounds. Such a linear relationship between pore density and drug flux is consistent with modelling presented in [10], and both Eq 2 and Eq 3. 

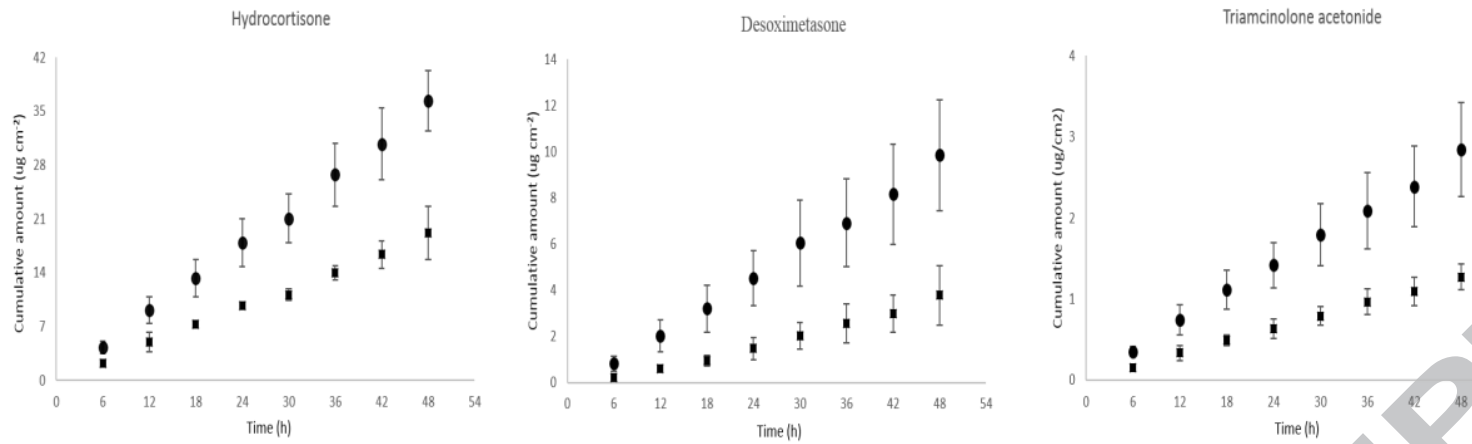

Fig.3. The plots of drug accumulation in the receptor phase during the experimental time. The squares and dots represent the amounts of drugs permeated through $1 \mathrm{x}$ rolled and 2 rolled skin, respectively.

Table 2 contains the values of variables relevant for both $\mathrm{Eq} 2$ and $\mathrm{Eq} 3$, and the values of the steady-state experimental drug fluxes and the fluxes predicted by the equations. The average density of created pores (over an area of about $1 \mathrm{~cm}^{2}$ ) was estimated at 52 pores $/ \mathrm{cm}^{2}$ for $1 \times$ rolled skin and twice as high for $2 \times$ rolled skin $\left(104\right.$ pores $\left./ \mathrm{cm}^{2}\right)$ (Fig. 2 (b) and (d), respectively). According to Fig.2, due to the unequal interspacing between microneedles on the microneedle roller (Fig.1), the horizontal interspacing between the pores on 1 x rolled skin was 1.5 times higher than the vertical interspacing. Further, the arrangement of pores on $2 \times$ rolled area appeared irregular due to the second roller application not being perfectly and consistently aligned with holes created by the first application. Therefore, in this study Eq 2 and Eq 3 will be applied for the scenarios when interspacing between the pores is irregular, which does not satisfy one of the assumptions made in deriving of the mathematical model [10]. This will test the applicability of the model and the equations for more realistic situations, as it is not always possible to achieve regular interspacing between the created pores, especially in the case of multiple application of a microporation technique over the same area of skin. The average values of $l_{p}$ for $1 \mathrm{x}$ rolled and $2 \mathrm{x}$ rolled skin were calculated in accordance with the pore density, to be applied for Eq 3 (Table 2). Further, as the pores created with microneedles usually had noncircular shape (Fig.2 (e)), as opposed to the pores created by laser ablation [13-16], an effective radius of the pore was used. The effective radius of the pores was estimated by adding a maximum longitudinal dimension of each pore to its average dimension in direction transverse to the longitudinal and dividing the obtained value by four. Thus, the average effective radius of the created pores was calculated to be $33.5 \mu \mathrm{m}$. The steady-state experimental drug fluxes were calculated from cumulative amount versus time slopes using all data points in Excel. 
Table 2. The values of experimental fluxes $\left(J_{\text {exp }}\right)$ of the corticosteroids through the skin treated with the microneedle roller and the related fluxes predicted by Eq $1\left(J_{\text {pred } 1}\right)$ and Eq $3\left(J_{\text {pred } 3}\right)$, and the parameters relevant for these equations as defined in Eq 1.

\begin{tabular}{|c|c|c|c|c|c|c|c|c|c|c|c|}
\hline Drug & $\begin{array}{l}M W \\
(\mathrm{Da})\end{array}$ & $\begin{array}{c}C_{v} \\
\left(\mu \mathrm{g} \mathrm{mL}^{-1}\right)\end{array}$ & $\begin{array}{c}n_{p} \\
\left(\mathrm{~cm}^{-2}\right)\end{array}$ & $\begin{array}{c}r_{p} \\
(\mu \mathrm{m})\end{array}$ & $\begin{array}{c}l_{p} \\
(\mathrm{~cm})\end{array}$ & $\begin{array}{c}D_{v}{ }^{1} \\
\left(\mathrm{~cm}^{2} \mathrm{~h}^{-1}\right)\end{array}$ & $K_{s}^{2}$ & $\begin{array}{c}D_{s}^{3} \\
\left(\mathrm{~cm}^{2} \mathrm{~s}^{-1}\right)\end{array}$ & $\begin{array}{c}J_{\text {exp }} \\
\left(\mu \mathrm{g} \mathrm{cm}^{-2} \mathrm{~h}^{-1}\right)\end{array}$ & $\begin{array}{c}J_{\text {predl }} \\
\left(\mu \mathrm{g} \mathrm{cm}^{-2} \mathrm{~h}^{-1}\right)\end{array}$ & $\begin{array}{c}J_{\text {pred } 3} \\
\left(\mu \mathrm{g} \mathrm{cm}^{-2} \mathrm{~h}^{-1}\right)\end{array}$ \\
\hline \multirow[t]{2}{*}{ Hydrocortisone } & \multirow[t]{2}{*}{362.5} & \multirow[t]{2}{*}{283.2} & 52 & \multirow[t]{6}{*}{33.5} & 0.14 & \multirow[t]{4}{*}{0.021} & \multirow[t]{2}{*}{2.21} & \multirow[t]{2}{*}{0.0014} & 0.39 & 0.52 & 0.48 \\
\hline & & & 104 & & 0.1 & & & & 0.75 & 1.03 & 0.96 \\
\hline \multirow[t]{2}{*}{ Desoximetasone } & \multirow[t]{2}{*}{376.5} & \multirow[t]{2}{*}{83.9} & 52 & & 0.14 & & \multirow[t]{2}{*}{9.38} & \multirow{2}{*}{0.00079} & 0.079 & 0.15 & 0.25 \\
\hline & & & 104 & & 0.1 & & & & 0.21 & 0.3 & 0.51 \\
\hline \multirow{2}{*}{$\begin{array}{c}\text { Triamcinolone } \\
\text { acetonide }\end{array}$} & \multirow[t]{2}{*}{434.5} & \multirow[t]{2}{*}{20.5} & 52 & & 0.14 & \multirow[t]{2}{*}{0.019} & \multirow{2}{*}{3.58} & \multirow{2}{*}{0.0012} & 0.029 & 0.034 & 0.041 \\
\hline & & & 104 & & 0.1 & & & & 0.058 & 0.067 & 0.082 \\
\hline
\end{tabular}

${ }^{1}$ The data for $D_{v}$ (equal to diffusion coefficient in water) was calculated in accordance with the previously developed mathematical model [10].

${ }^{2}$ The data for $K_{s}$ was calculated in accordance with the solubility of the corticosteroids in dermis reported in the paper by Anissimov and Roberts [11], and the corresponding values of solubility in the donor solution $\left(=C_{v}\right)$.

${ }^{3}$ The data for $D_{s}$ (assumed equal to the diffusion coefficient in dermis) was obtained from the paper by Anissimov and Roberts [11]. 
The results of predicted fluxes in Table 2 demonstrate generally excellent prediction of the drug fluxes with both equations, Eq $2\left(J_{\text {pred1 }}\right)$ and Eq $3\left(J_{\text {pred } 2}\right)$. Eq 2 gives a relatively higher accuracy of predictions for desoximetasone compared to the predictions by Eq 3. The better results by the simple equation are probably a reflection in uncertainty of determining parameters for the Eqs 2 and 3, such as the average radius. Fig.4 highlights the strong correlation between the fluxes predicted with the simple Eq 2, and experimental fluxes from the data collected in the study by Rzhevskiy et al. [10] and Table 2. Interestingly, the accuracy of predictions of drug fluxes across $1 \times$ rolled and $2 \times$ rolled skin is relatively similar, while it was expected that the more irregular interspacing between pores in $2 \times$ rolled skin (Fig. 2 (c)) should have led to a poorer accuracy.

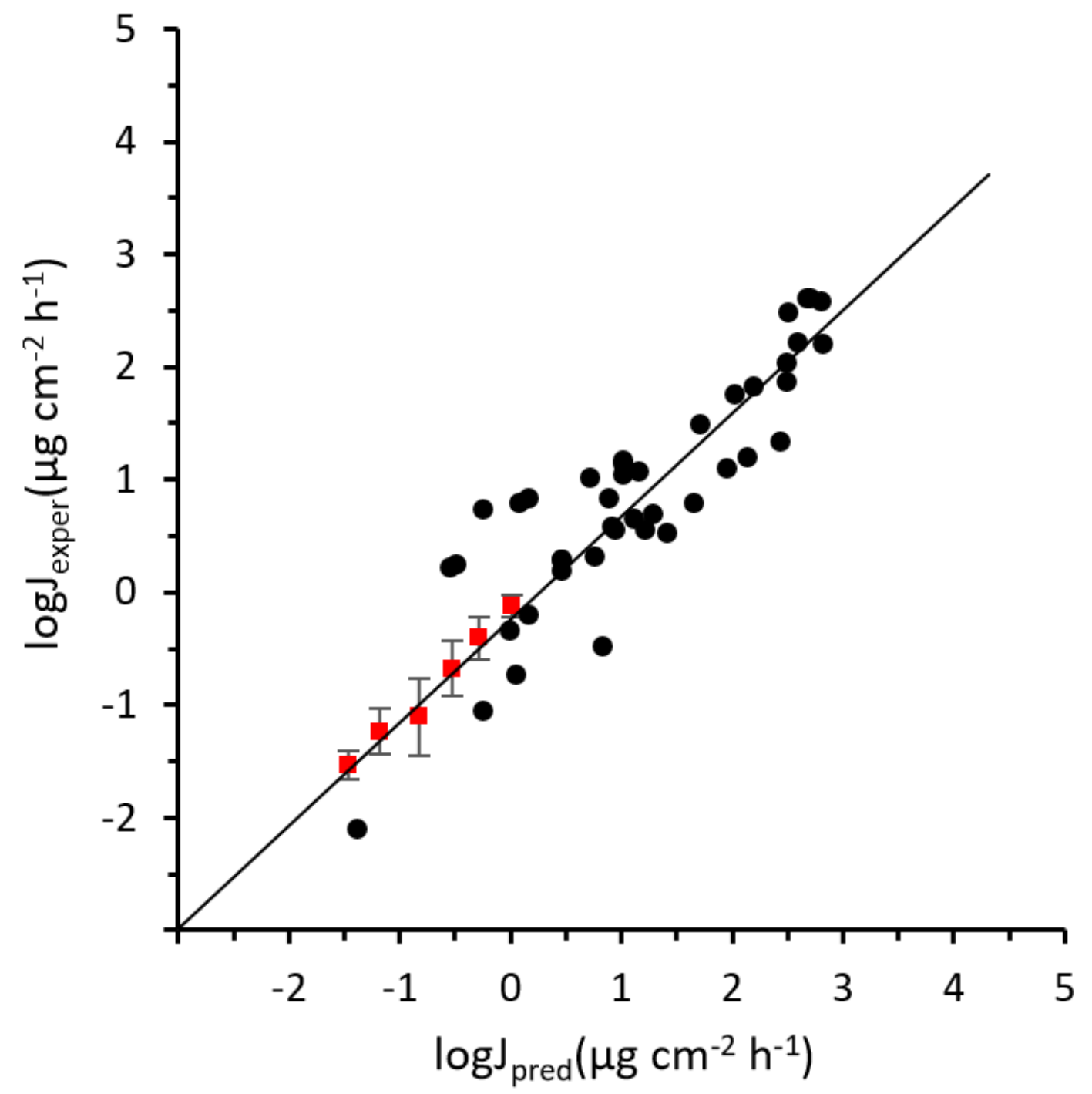

Fig.4. The values of fluxes predicted by Eq 2 vs the values of the experimental fluxes from Table 2 (red squares), and the data collected in the paper by Rzhevskiy et al. [10] (black circles). 
Thus, the lack of regular interspacing between created pores does not appear to influence flux predictions with Eqs 2 and 3, demonstrating a robustness and high flexibility of the model in application to practical cases of microneedle treated skin.

Although all three solutes investigated in this work were lipophilic (with $\log P$ of 1.6, 2.3 and 2.5 for Hydrocortisone, Desoximetasone and Triamcinolone acetonide respectively) the flux through the skin for all of them was significantly enhanced by microporation. This enhancements demonstrates that though the SC barrier has lipophilic pathways that would, intuitively thinking, favour the penetration of lipophilic solutes, the aqueous pathways created by microporation result in a much higher flux. The explanation to this apparent contradiction is twofold. Firstly, as was previously demonstrated [17], lipophilicity plays a relatively minor role in determining the maximum flux of solutes through skin, distinct to a strong dependence of a permeability coefficient (a less physiological parameter) on lipophilicity. Secondly and most importantly, the diffusion coefficient in the viable skin is many orders of magnitude higher than the effective diffusion coefficient in the SC, so that even lipophilic solutes with relatively poor aqueous solubility have a very significant flux through large aqueous pathways created by microporation.

\section{Statistical validation of the simple equation by regression analysis}

Table 3 represents the results of five regression analyses, using equation (4) for two different scenarios:

1. When only parameter $A$ is varied and powers $b, c$ and $d$ are all fixed.

2. When parameter $A$ is varied together with one of the powers $(b, c$ or $d$ ), while other two powers are fixed.

Table 3. The results of the regression analyses

\begin{tabular}{|c|c|c|c|c|c|c|}
\hline & Sum of squared deviations & $\mathrm{R}^{2}$ & $A$ & $b$ & $c$ & $D$ \\
\hline Original parameter values & 14.6 & 0.819 & 0.36 & 1 & 0.6 & 1 \\
\hline Only $A$ is varied & 12.31 & 0.847 & 0.22 & 1 & 0.6 & 1 \\
\hline$A$ and $b$ are varied & 12.02 & 0.851 & 0.079 & 0.82 & 0.6 & 1 \\
\hline$A$ and $c$ are varied & 11.29 & 0.860 & 0.068 & 1 & 0.44 & 1 \\
\hline$A$ and $d$ are varied & 12.26 & 0.848 & 0.321 & 1 & 0.6 & 0.93 \\
\hline
\end{tabular}

The values in Table 3 demonstrate that the best improvement, compared to the unmodified, theoretically derived, parameters is achieved when $A$ is varied, as $\mathrm{R}^{2}$ increased from 0.819 to 0.847 . There is only small additional improvement when powers $b, c$ or $d$ are allowed to vary (a further increase in $\mathrm{R}^{2}$ from 0.847 to a maximum of 0.860 when the power of $M W, c$, is varied). 
Further, the coefficients do not deviate very far from their theoretical values: $b$ from 1 to $0.82, c$ from 0.6 to 0.44 and $d$ from 1 to 0.93 . This regression analysis further supports the derived equations and indicates that further meaningful improvement is possible when only constant $A$ is adjusted.

A regression with all the parameters varying was also performed. Although there was a noticeable improvement in $\mathrm{R}^{2}$ from 0.860 (when $A$ and $c$ are varied) to 0.897 , the parameter values were not reasonable $(b=0.091, c=0.26$ and $d=0.42)$. Such sluggish dependence of the flux on the radius of pores $\left(r_{p}^{0.091}\right)$, their density $\left(n_{p}^{0.42}\right)$ and molecular weight $\left(M W^{-0.26}\right)$ is not reasonable and is most likely the result of using too many regression parameters (four) on too few data points (49). Further contributing to the poor outcome when regressing all parameters may be the correlation of parameters with each other.

\section{Practical application of the simple equation}

In general, it can be concluded that the simple Eq 2 gives a good prediction, and is generally more practical than the complex Eq 3. Therefore, Eq 2 is potentially applicable to the estimation of drug delivery through microporated skin in practical cases of drugs which do not bind or metabolize within the skin to a significant degree, and are delivered using an aqueous drug vehicle. Eqs 2 and 3 can be applied to any case where micropores are produced in the skin [10], therefore as a model case, the prediction of the delivery of methotrexate (MTX) through the skin microporated with erbium: yttrium aluminum garnet (Er:YAG; $2940 \mathrm{~nm}$ ) laser will be investigated.

MTX belongs to the group of folate antagonists, and has anti-inflammatory and chemotherapeutic properties [18]. One of the major uses of MTX is the treatment of localised rheumatic disorders, and rheumatism in particular. Anti-rheumatic treatment with MTX is usually performed via parenteral or oral administration routes with a relatively low bioavailability, using high doses of the drug. However, such systemic MTX administration is associated with several adverse effects, and therefore topical administration is preferable as it may potentially minimize possible complications.

Originally, topical treatment of rheumatism with MTX was considered ineffective, due to the low permeability of the drug through intact skin. In a recent in vitro study by Taudorf et al., skin microporation with a fractional $2940 \mathrm{~nm}$ Er:YAG laser was shown to be effective in enhancing topical MTX delivery [19]. In this study, the laser created round-shaped micropores in full- 
thickness porcine skin at an average radius of $137.5 \mu \mathrm{m}$ and a density of $47 \mathrm{pores} / \mathrm{cm}^{2}$. The experiments were conducted in Franz cells with the diffusion area at $0.64 \mathrm{~cm}^{2}$, at $37{ }^{\circ} \mathrm{C}$, using microporated and intact skin. A $10 \mathrm{mg} / \mathrm{ml}$ MTX solution, prepared by appropriately diluting Metex ${ }^{\circledR} 50 \mathrm{mg} / \mathrm{ml}$ aqueous solution for injection with $7.39 \mathrm{pH}$ PBS was applied as a donor and PBS was used as a receptor. In the experiments with microporated skin, the average concentration of MTX in the receptor was found to be $1.25 \mathrm{mg} / \mathrm{ml}$ after $24 \mathrm{~h}$, while in the experiments with intact skin the average concentration of MTX in the receptor was $0.006 \mathrm{mg} / \mathrm{ml}$ after $21 \mathrm{~h}$. Thus, skin microporation provided approximately 200 times higher transdermal delivery of MTX compared to the MTX delivery through intact skin [19].

The average experimental transdermal flux of MTX through the microporated skin was $55.6 \mu \mathrm{g}$ $\mathrm{cm}^{-2} \mathrm{~h}^{-1}$ (calculated in accordance with the amount of drug in the receptor after 24 hours of the experiment, considering the first 1.5 hours as a lag time). Using Eq 2 the predicted MTX flux was $59.2 \mu \mathrm{g} \mathrm{cm}^{-2} \mathrm{~h}^{-1}$ (calculated in accordance with the values: $M W$ at $454 \mathrm{Da}, r_{p}$ at $0.01375 \mathrm{~cm}$, $n_{p}$ at $47 \mathrm{~cm}^{-2}$ and $C_{v}$ at $10000 \mu \mathrm{g} / \mathrm{ml}$ [19]), a $6.5 \%$ difference from the experimental value. Therefore in this case, Eq 2 can be reliably applied to estimate the transdermal drug flux in vitro. Further, Eq 2 can assist in an estimation of the accumulation of the drug in the underlying tissues for an in vivo model, if the rate of drug clearance from the tissues is appropriately modelled. For instance, as the drug flux through microporated skin has a linear dependence on the radius and density of created pores, the two times increase in one of these variables is required to achieve the twice higher transdermal drug delivery. Consequently, Eq 2 can be used in the design, prediction and validation of the results obtained in vitro and in vivo, particularly in the investigation of rheumatism treatment via microporation-enhanced transdermal MTX delivery.

\section{CONCLUSION}

In this study, we measured experimental fluxes of three corticosteroids through microporated human skin to assess the validity of previously derived equations. The results indicated that both simple and complex equations gave good approximation. However, the simple equation (Eq 2) was preferred to the complex equation (Eq 3), as it was more convenient and contained fewer experimentally-determined variables, which may be difficult to obtain for some drugs. A good agreement between the predicted and experimental fluxes was achieved in this work in spite of irregular interspacing between the pores and their non-circular shape, which was nor previously confirmed for the equations [10]. Such a feature highlights the robustness and flexibility of the equations and underlines their applicability for the design of in vitro and in vivo experiments. 
The simple equation form was also confirmed by regression analysis, indicating that the literature data supports its current form. To further validate and exemplify the use of the simple equation, it was applied to predict experimental microporation-enhanced MTX flux which was compared to the literature data. The results indicated a high accuracy of prediction, which suggested it can be used for insights for analysing and planning in vivo experiments and clinical investigations for the treatment of localised rheumatic disorders via microporation-enhanced transdermal MTX delivery. At this stage, the prediction of drug flux through microporated skin with simple equation is confirmed for aqueous vehicles and when a drug does not metabolize or bind in skin. Further work is required to extend the model to a wider class of drugs and more general application conditions.

\section{References}

[1] C.R. Harding, The stratum corneum: structure and function in health and disease, Dermatologic therapy, 17 (2004) 6-15.

[2] O.G. Jepps, Y. Dancik, Y.G. Anissimov, M.S. Roberts, Modeling the human skin barrier - Towards a better understanding of dermal absorption, Adv Drug Deliv Rev, 65 (2013) 152-168.

[3] K. Ita, Transdermal drug delivery: progress and challenges, Journal of Drug Delivery Science and Technology, 24 (2014) 245-250.

[4] S. Wiedersberg, R.H. Guy, Transdermal drug delivery: 30+ years of war and still fighting!, Journal of Controlled Release, 190 (2014) 150-156.

[5] M.R. Prausnitz, R. Langer, Transdermal drug delivery, Nature biotechnology, 26 (2008) 1261-1268.

[6] T.R.R. Singh, R.F. Donnelly, Microporation in Penetration Enhancement, in: Percutaneous Penetration Enhancers Physical Methods in Penetration Enhancement, Springer, 2017, pp. 257-271.

[7] A.K. Banga, Microporation applications for enhancing drug delivery, Expert Opinion on Drug Delivery, 6 (2009) 343-354.

[8] H. Kalluri, A.K. Banga, Formation and closure of microchannels in skin following microporation, Pharmaceutical research, 28 (2011) 82-94.

[9] R.F. Donnelly, T.R. Raj Singh, A.D. Woolfson, Microneedle-based drug delivery systems: microfabrication, drug delivery, and safety, Drug Deliv, 17 (2010) 187-207.

[10] A.S. Rzhevskiy, R.H. Guy, Y.G. Anissimov, Modelling drug flux through microporated skin, Journal of Controlled Release, 241 (2016) 194-199.

[11] Y.G. Anissimov, M.S. Roberts, Modelling dermal drug distribution after topical application in human, Pharmaceutical research, 28 (2011) 2119-2129.

[12] V.R. Leite-Silva, W.Y. Sanchez, H. Studier, D.C. Liu, Y.H. Mohammed, A.M. Holmes, E.M. Ryan, I.N. Haridass, N.C. Chandrasekaran, W. Becker, J.E. Grice, H.A.E. Benson, M.S. Roberts, Human skin penetration and local effects of topical nano zinc oxide after occlusion and barrier impairment, Eur J Pharm Biopharm, 104 (2016) 140-147.

[13] Y. Bachhav, S. Summer, A. Heinrich, T. Bragagna, C. Böhler, Y. Kalia, PLEASE ${ }^{\circledR}$ A promising tool for intraepidermal drug delivery, in: Annual Meeting of the Controlled Release Society, 2008.

[14] Y. Bachhav, S. Summer, A. Heinrich, T. Bragagna, C. Böhler, Y. Kalia, Effect of controlled laser microporation on drug transport kinetics into and across the skin, Journal of Controlled Release, 146 (2010) 31-36. 
[15] Y.G. Bachhav, A. Heinrich, Y.N. Kalia, Using laser microporation to improve transdermal delivery of diclofenac: increasing bioavailability and the range of therapeutic applications, European Journal of Pharmaceutics and Biopharmaceutics, 78 (2011) 408-414.

[16] Y. Bachhav, A. Heinrich, Y. Kalia, Controlled intra-and transdermal protein delivery using a minimally invasive Erbium: YAG fractional laser ablation technology, European Journal of Pharmaceutics and Biopharmaceutics, 84 (2013) 355-364.

[17] B.M. Magnusson, Y.G. Anissimov, S.E. Cross, M.S. Roberts, Molecular size as the main determinant of solute maximum flux across the skin, Journal of Investigative Dermatology, 122 (2004) 993-999.

[18] P. Ranganathan, S. Eisen, W. Yokoyama, H. McLeod, Will pharmacogenetics allow better prediction of methotrexate toxicity and efficacy in patients with rheumatoid arthritis?, Annals of the rheumatic diseases, 62 (2003) 4-9.

[19] E.H. Taudorf, C.M. Lerche, A.-C. Vissing, P.A. Philipsen, J. Hannibal, J. D'Alvise, S.H. Hansen, C. Janfelt, U. Paasch, R.R. Anderson, Topically applied methotrexate is rapidly delivered into skin by fractional laser ablation, Expert opinion on drug delivery, 12 (2015) 1059-1069. 
Graphical abstract

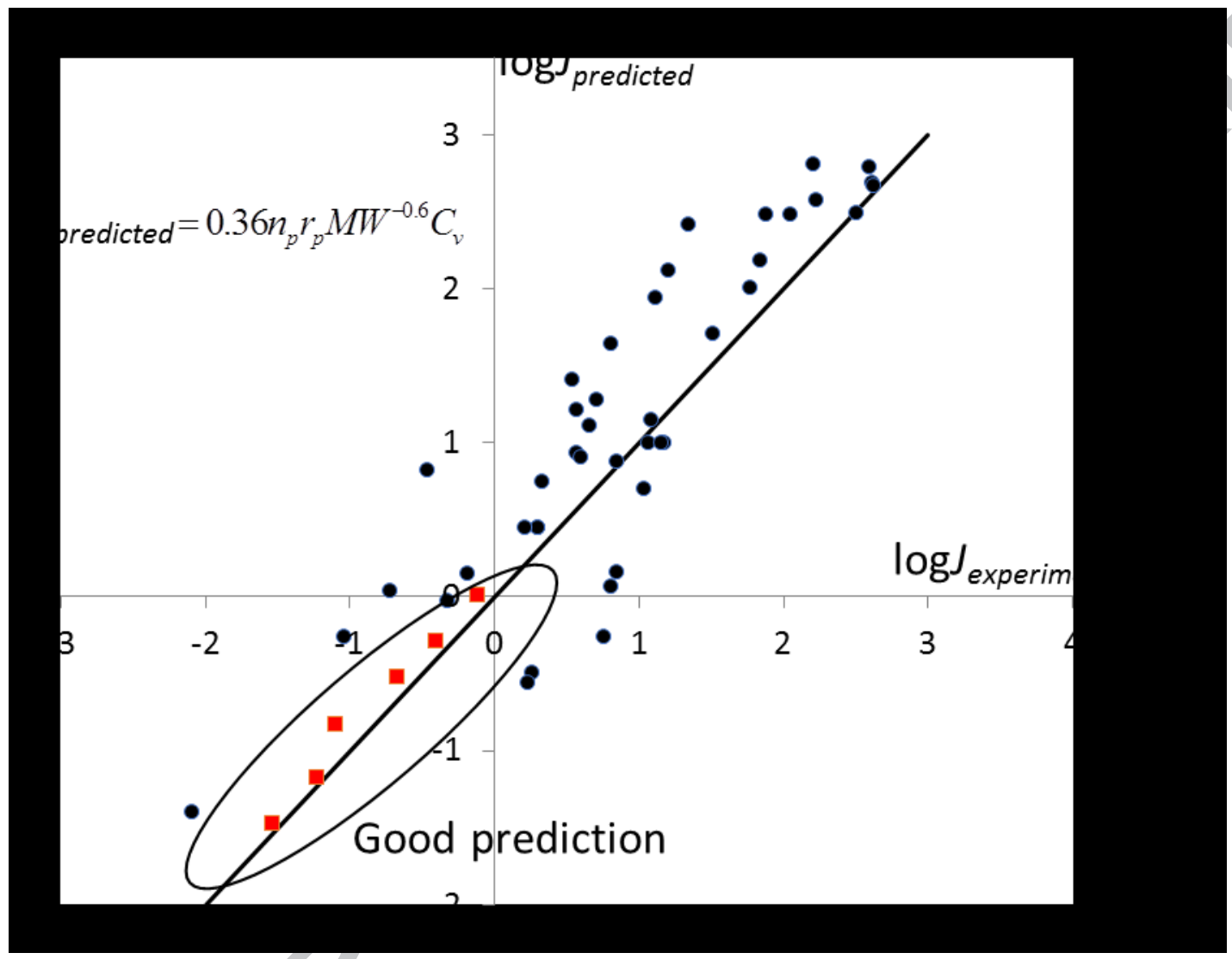




\section{Accepted Manuscript}

Research paper

Jurepean

lourinal of

Pharmaceuties and

Biepharmaceutics

Using a simple equation to predict the microporation-enhanced transdermal drug flux

Alexey S. Rzhevskiy, Krishna Telaprolu, Yousuf H. Mohammed, Jeffrey E.

Grice, Michael S. Roberts, Yuri G. Anissimov

PII:

S0939-6411(17)31228-6

DOI:

https://doi.org/10.1016/j.ejpb.2018.01.019

Reference:

EJPB 12679

To appear in:

European Journal of Pharmaceutics and Biopharmaceutics

Received Date:

24 October 2017

Revised Date:

30 January 2018

Accepted Date:

30 January 2018

Please cite this article as: A.S. Rzhevskiy, K. Telaprolu, Y.H. Mohammed, J.E. Grice, M.S. Roberts, Y.G. Anissimov, Using a simple equation to predict the microporation-enhanced transdermal drug flux, European Journal of Pharmaceutics and Biopharmaceutics (2018), doi: https://doi.org/10.1016/j.ejpb.2018.01.019

This is a PDF file of an unedited manuscript that has been accepted for publication. As a service to our customers we are providing this early version of the manuscript. The manuscript will undergo copyediting, typesetting, and review of the resulting proof before it is published in its final form. Please note that during the production process errors may be discovered which could affect the content, and all legal disclaimers that apply to the journal pertain. 